\title{
COMPACT SEMIGROUPS WITH LOW DIMENSIONAL ORBIT SPACES
}

\author{
R. P. HUNTER
}

ABSTRACr. If $S$ is a compact connected semigroup, $G$ a compact subgroup at the identity such that $S / G$ is either two dimensional or embeddable in three space, then the normalizer conjecture holds.

Let $S$ be a compact connected monoid and $G$ be a compact connected subgroup at the identity. If the orbit space $S / G$ is either one dimensional or planar then the orbits form a congruence [1], [2]. In particular, a local thread lies in the centralizer of $G$. Thus, the centralizer conjecture holds for such semigroups [6].

We note here that if $S / G$ is of dimension two or is embeddable in three space then the centralizer conjecture (=normalizer conjecture [3]) holds.

Proposition. Let $S$ be a compact connected monoid and let $G$ be a compact connected subgroup at the identity. If the orbit space $S / G$ is either of dimension two or is embeddable in three space then $S$ contains a compact connected subsemigroup $F$ such that $F$ contains $G$, the orbits of $G$ form a congruence in $F$, and $F$ meets the minimal ideal of $S$.

Indication of Proof. Let us suppose first that $S / G$-the space of orbits $\{x G\}$-has dimension less than or equal to two. Letting $G$ act on the left on $S / G$ by $g \bar{x}=g\{x G\}=\{g x G\}$, we first note that each stability subgroup $G_{\bar{x}}$ is normal and that each quotient group $G / G_{\bar{x}}$ is trivial or is one dimensional. In effect, let $L$ be the semisimple part of $G$ and consider the quotient space $\tilde{L}(\bar{x})=L / G_{\bar{x}} \cap L$. If $\operatorname{dim} \tilde{L}(\bar{x})=0$ then, of course, $G_{\bar{x}}$ contains $\tilde{L}(\bar{x})$. Next, we notice that $\operatorname{dim} \tilde{L}(\bar{x})$ cannot be one [6], [8]. Were we to have $\operatorname{dim} \tilde{L}(\bar{x})=2$, we could apply an argument of Wallace. [4] to the action of $S$ upon $S / G$ to show that $H^{2}(\tilde{L}(\bar{x}), Z)=0$. However, since $\tilde{L}(\bar{x})$ is a two manifold, this is impossible. Thus, in any case, $G_{\bar{x}}$ contains $L$ and is consequently normal.

Finally, $G / G_{\bar{x}}$ cannot have dimension two since again Wallace's argument would show that $H^{2}\left(G / G_{\bar{x}}, Z\right)=H^{2}(G \bar{x}, Z)=0$. However, a two dimensional compact connected group $T$ cannot be such that $H^{2}(T, Z)$ is

Received by the editors July 18, 1972 and, in revised form, November 19, 1972.

AMS (MOS) subject classifications (1970). Primary 22A15.

Key words and phrases. Compact semigroup, dimension, cohomology.

(c) American Mathematical Society 1973 
trivial. Thus, $G / G_{\bar{x}}$ is either trivial or is a one dimensional compact connected (abelian) group. Indeed, since each $G_{\bar{x}}$ contains $[G, G]$, the action of $G$ can be factored through $G /[G, G]$ :

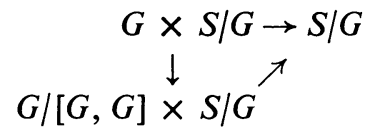

We now have an abelian group $G /[G, G]$ acting on the acyclic space $S / G$. (At this point, we may, without loss of generality, assume $S$ has a zero, considering $S / K$ if necessary.) The set of fixed points $F^{\prime}$ in $S / G$ under the action of $G /[G, G]$ is connected [6]. The pre-image $F$ of $F^{\prime}$ back in $S$ is the desired compact connected semigroup in which $G$ is (left) normal.

Suppose now that $S / G$ is embeddable in three space. With the notation of the first part of the argument, we note that if $x \notin K$ then $\operatorname{dim} \tilde{L}(\bar{x})=0$. As in the first part, we know that $\operatorname{dim} \tilde{L}(\bar{x}) \neq 1$. We certainly cannot have $\operatorname{dim} \tilde{L}(\bar{x})=3$. Assume then, that $\operatorname{dim} \tilde{L}(\bar{x})=2$. First, let $\overline{1}$ belong to the unbounded complementary domain of $\tilde{L}(\bar{x})$. In this case $K / G$, since it carries the cohomology of $S / G$, must lie in the bounded complementary domain of $\tilde{L}(\bar{x})$. Since the orbit of $\overline{1}$ is degenerate, we cannot have points $\bar{x}$ arbitrary close to $\overline{1}$ with $\operatorname{dim} G \bar{x}=2$. We must then have some $\tilde{L}(\bar{t})$ of dimension two such that, given any $\bar{y}$ in its unbounded complementary domain, $\operatorname{dim} G \bar{y}<2$. But this would entail having a sequence of orbits of dimension zero or one converging to a two dimensional orbit, which is impossible.

Lastly, if $\overline{1}$ is in the bounded complementary domain of $\tilde{L}(\bar{x})$, one can proceed, as in [2], to show that $S / G$ must contain this domain. However, this would mean that $S / G$ contains a Euclidean neighborhood about $\overline{1}$. This is impossible [5], [7].

Thus, as in the first part, $G_{\bar{x}}$ must contain $[G, G]$ and the previous considerations apply again.

\section{REFERENCES}

1. L. W. Anderson and R. P. Hunter, Compact semigroups having certain one dimensional hyperspaces, Amer. J. Math. 92 (1970), 894-896. MR 43 \#2149.

2. - A remark on compact semigroups having certain decomposition spaces embeddable in the plane, Bull. Austral. Math. Soc. 4 (1971), 137-139; Corrigendum, ibid. 4 (1971), 432. MR 42 \#7815; MR 43 \#6357.

3. K. Hofmann and M. Mislove, The centralizing theorem for left normal groups in compact monoids, Semigroup Forum 3 (1970), 31-42.

4. A. D. Wallace, Cohomology dimension and mobs, Summa Brasil. Math. 3 (1953), 43-55. MR 15, 336. 
5. A. D. Wallace, The Gebietstreue in semigroups, Nederl. Akad. Wetensch. Proc. Ser. A 59=Indag. Math. 18 (1956), 271-274. MR 18, 14.

6. K. Hofmann and P. Monstert, Elements of compact semigroups, Merrill, Columbus, Ohio, 1966. MR 35 \#285.

7. D. Stadtlander, Actions with topologically restricted state space. II, Duke Math. J. 38 (1971), 7-14. MR 42 \#7822.

8. J. Tits, Sur une classe de groupes de Lie resolubles, Bull. Soc. Math. Belg. 11 (1959), 100-115. MR 22 \#11063.

Department of Mathematics, Pennsylvania State University, University Park, Pennsylvania 16802 